\title{
Germanica
}

\section{Philistins, bâtisseurs et psychopathes : les Caïn et Abel de Borngräber, Fuhrmann et Koffka}

Philister, Erbauer und Psychopathen: Kain und Abel bei Borngräber, Fuhrmann und Koffka

\section{Gérard Laudin}

\section{(2) OpenEdition}

\section{Journals}

Édition électronique

URL : http://journals.openedition.org/germanica/2247

DOI : 10.4000/germanica.2247

ISSN : 2107-0784

\section{Éditeur}

Université de Lille

Édition imprimée

Date de publication : 1 janvier 1999

Pagination : 13-28

ISBN : 2-913857-00-0

ISSN : 0984-2632

\section{Référence électronique}

Gérard Laudin, «Philistins, bâtisseurs et psychopathes : les Caïn et Abel de Borngräber, Fuhrmann et Koffka », Germanica [En ligne], 24 | 1999, mis en ligne le 11 octobre 2013, consulté le 06 octobre 2020 URL : http://journals.openedition.org/germanica/2247 ; DOI : https://doi.org/10.4000/germanica.2247

Ce document a été généré automatiquement le 6 octobre 2020.

(c) Tous droits réservés 


\section{Philistins, bâtisseurs et psychopathes : les Caïn et Abel de Borngräber, Fuhrmann et Koffka}

Philister, Erbauer und Psychopathen: Kain und Abel bei Borngräber, Fuhrmann und Koffka

Gérard Laudin

1 L'histoire de Caïn et Abel fait partie des thèmes bibliques les plus souvent abordés dans les œuvres littéraires. Publiés entre 1908 et 1917, les textes d'Otto Borngräber, Paul Fuhrmann et Friedrich Koffka prolongent une longue série de mutations qui ont affecté très tôt le traitement du thème de Caïn dans la littérature allemande. Parfois détaché dès le milieu du XVIII siècle de ses principales références religieuses, voire intégré à des contextes résolument profanes, il est progressivement investi de préoccupations et valeurs sociales, les relations familiales et la puissance paternelle. Une première rupture importante avec la compréhension patristique et manichéenne traditionnelle est amorcée avec Der Tod Abels de Salomon Geßner (1758) qui, bientôt suivi de Friedrich et Meta Klopstock et de Maler Müller, peignit en Caïn un être mélancolique, sombre, introverti, plus malheureux que mauvais. Ils réalisent ainsi une réhabilitation partielle de Caïn et font de lui un archétype d'humanité souffrante ${ }^{1}$. Le Caïn (1821) de Lord Byron ajoutera à cette perspective la représentation d'un Caïn déchiré et malheureux parce qu'assoiffé de connaissances inaccessibles, tout en esquissant une autre évolution radicalement nouvelle : la dévalorisation du personnage d'Abel, amplifiée plus tard par Paul Fuhrmann et Friedrich Koffka qui feront de lui un être dérisoire et vulgaire, superficiel et insignifiant.

2 Ces différentes modifications du thème primitif, en particulier les inversions au moins partielles de valeur entre Caïn et Abel, se retrouvent chez les nombreux auteurs qui dans les années 1880-1920 s'intéressent de nouveau à ce thème largement délaissé en Allemagne depuis une centaine d'années ${ }^{2}$. On perçoit dans certains textes une influence nietzschéenne, qu'on retrouvera aussi chez Borngräber et Fuhrmann, par exemple dans Die Bücher Kains vom ewigen Leben (1899) d'Eduard von Meyer où Caïn aboutit à la 
conclusion que Dieu n'existe que dans l'imagination des hommes et que le christianisme est haine de la vie. D'autres textes continuent de se situer plus nettement dans l'héritage byronien, soit qu'ils redonnent, comme le poème épique de Gustav Kastropp ${ }^{3}$, au motif de l'amour la place qu'il avait perdue chez Byron, soit qu'ils empruntent au poète anglais, comme le fait Ludwig Weber ${ }^{4}$, l'idée d'un Abel obéissant et superficiel, alternativement doux et colérique $(W, 130)$. Un thème nouveau, commun aux textes de Kastropp et Weber, et qu'on retrouvera chez Borngräber, Fuhrmann et Koffka, est le dégoût exprimé par Caïn à l'égard des sacrifices d'animaux. Chez Byron déjà, Caïn est horrifié à l'idée que Dieu puisse trouver du plaisir au bêlement douloureux des mères qui gémissent sur leurs nourrissons égorgés (III, 1, v. 298-301). $\mathrm{Au}$ XVIII ${ }^{\mathrm{e}}$ siècle déjà, Herder interprétait le passage de la cueillette et de l'agriculture à la chasse comme un signe de décadence de la civilisation, car le sang des animaux avait rendu l'homme sauvage ${ }^{5}$. Rapidement évoqué par Kastropp (K, 15-18,195, 269), ce motif est repris par Weber qui en fait un des axes essentiels du conflit entre les deux frères: Caïn demande à Abel s'il peut supporter le regard de l'agneau qu'il va tuer; pour Caïn, le sacrifice rituel n'est qu'un acte de cruauté, et si Dieu l'agrée, c'est qu'il est cruel luimême $(\mathrm{W}, 37)$. Le regard de l'animal qui demande : pourquoi ? devient une métaphore de l'injustice gratuite. Elle occupera chez Fuhrmann une place centrale et sera chez Koffka la cause immédiate du meurtre.

3 Plusieurs fois rééditée, la pièce Die ersten Menschen. Erotisches Mysterium (Berlin, 1908) d'Otto Borngräber 6 , qui est l'auteur d'autres textes d'inspiration religieuse et/ou erotique ainsi que d'une thèse sur la philosophie de la Réforme, présente trois innovations essentielles: l'action, y compris le fratricide, découle intégralement des désirs érotiques des personnages; Dieu n'intervient à aucun moment; les rapports pédagogiques traditionnels entre parents et enfants sont inversés.

Il était habituel dans les textes postérieurs à Geßner que des relations amoureuses existent entre Caïn, Abel et leurs sœurs. Borngräber substitue à cet amour aux contours flous un érotisme bien charnel, et l'action, qui ne compte qu'un personnage féminin, se déroule dans un monde qui, ignorant les tabous, pratique l'inceste en toute innocence ${ }^{7}$. Borngräber peut avoir trouvé dans des travaux ethnologiques modernes, qui ont inspiré à Freud certaines analyses de Totem und $T a b u^{8}$, l'idée de relier le mythe des origines de l'humanité à un motif fréquent dans la littérature des années 1900-1920 : la rivalité jalouse sur fond de désirs incestueux entre frère et sœur, mais aussi entre mère et fils ${ }^{9}$. Otto Rank interprète la pièce de Borngräber comme la rivalité entre les frères pour obtenir la mère ${ }^{10}$.

Borngräber a voulu thématiser des questions philosophiques. L'attitude de Kajin s'inscrit dans une vaste recherche hédoniste du plaisir terrestre: «Ich will [...] austrinken die Welt» $(B, 25)$. Cet hédonisme, qui est d'autant plus fort que Kajin est malheureux (B,15), supplante la quête de la connaissance en tant que tentative de parvenir au bonheur, à un bonheur purement terrestre. Toutefois, le propos de Borngräber n'est pas dépourvu d'ambiguïtés. Dans la sensualité de Chawa, Adahm ne voit que la superficialité et la stupidité d'un être pauvre parce qu'incapable de s'élever comme l'homme à la spiritualité $(\mathrm{B}, 10,19)$. Mais, d'une façon contradictoire, Adahm déclare également que l'Éden, c'était "als ich war, was Chawa - blieb» (B, 16). Borngräber veut-il dénoncer la misogynie d'Adahm ou cherche-t-il à démythifier l'Eden ? Sans doute faut-il comprendre qu'il suggère, dans une perspective kantienne, que l'hominisation est un processus qui éloigne progressivement l'homme de 
l'animalité. Cette idée est métaphorisée par ce qui constitue chez Borngräber une innovation importante : l'inversion des rapports d'éducation traditionnels à l'intérieur de la famille d'Adam. Chez Borngräber, Adahm pense que Chabel tend intuitivement, malgré son jeune âge, vers une sagesse que lui-même n'a acquise que très tard (B, 19). Ce n'est pas Adahm, mais Chabel, représentant de la "seconde génération», qui accomplit un progrès décisif : c'est lui qui, soudain, découvre l'idée de Dieu qu'Adahm jusqu'alors ignorait après l'avoir recherchée toute sa vie (B,20), avant d'apporter progressivement la révélation des attributs de Dieu : «allmächtig - allweise - allgütig» (B, 27).

6 L'intention de Borngräber fut peut-être d'écrire une pièce à la fois naturaliste et symboliste, mais il n'est pas sûr qu'il ait perçu que la représentation concrète de cette animalité des premiers hommes produisait un effet burlesque: quand par exemple Caïn, dont on perçoit le «wildes Schnaufen» $(B, 47)$, traverse la scène comme un animal en rut et s'écrie: «Finden will ich das wilde wilde Weib!» $(\mathrm{B}, 46)$ ou bien quand il annonce sans détour à sa mère qu'il s'apprête à déshabiller « d'une manière bestiale » : «Ich begehre dein!» (B, 22).

7 Il semble surtout que Borngräber vise à détruire, dans une intention peut-être antichrétienne, les croyances relatives aux origines. Peu après avoir découvert l'idée de Dieu, Chabel est assailli par le désir physique et commet l'inceste avec Chawa. Plus tard, Kajin dira que Dieu est un fantôme issu de l'imagination de son frère $(B, 69)$ et que la religion est fuite devant la vie (B, 53). Kajin a affirmé que s'il existe un Dieu, c'est un deus sive natura qu'il faut créer, afin de pouvoir se rendre Dieu soi-même : l'homme en lutte deviendrait un dieu vivant $(B, 60)$. Le fait que Chabel déclare avant de mourir que Dieu lui a donné la vie au moment où il se trouvait dans les bras de Chawa $(\mathrm{B}, 64)$ suggère que la foi et la spiritualité procèdent de la sublimation de désirs; le fait que Dieu ne se soit jamais manifesté et qu'on le découvre pour la première fois dans les propos quelque peu illuminés de Chabel, qui était «comme un somnambule» $(B, 18)$ quand il fit cette découverte, tendrait à lui dénier toute existence hors de l'imagination des hommes. Il est dit d'ailleurs clairement que Chabel perçut l'éternité et la nomma Dieu $(B, 20)$. La perspective est nominaliste. La prétendue pureté originelle se réduit en animalité et la spiritualité qui lui succède paraît n'être qu'un assemblage de fantasmes.

8 L'idée d'inversion des rôles pédagogiques se retrouve en 1909 dans la tragédie de Paul Fuhrmann ${ }^{11}$, dont l'Adam reconnaît à Caïn une valeur peut-être supérieure à la sienne $(F, 18)$ et susceptible de faire de lui le rédempteur de la famille. Mais Adam souffre de voir son fils s'engager sur le chemin où lui-même a échoué, dans une recherche de l'absolu qui lui paraît mener hors de la vie $(F, 18)$. En fait, en tentant de reconstruire l'Éden, Caïn va rechercher un absolu qu'il veut situer dans la vie quotidienne des siens.

9 Dès la première scène de la pièce, Caïn se montre horrifié par le sacrifice $(F, 2)$ et par le spectacle de la mort. Mais parallèlement à cet argument maintenant traditionnel, Caïn révèle par cette attitude un certain pragmatisme utilitariste: le sacrifice est une absurdité anti-économique, surtout en période d'épidémie. Il en déduit que le sacrifice n'est pas une exigence imposée par Dieu, mais dictée à Adam par les puissances du mal qui l'habitent $(F, 3)$. Certaines prétendues exigences divines seraient donc en fait de l'idéologie humaine. De plus, Caïn remarque qu'Adam non seulement sacrifie des animaux mais aussi poursuit de sa haine des hommes qui lui ressemblent en tous points mais à qui il reproche de ne pas avoir connu le paradis (F,4). Au motif de l'animal sacrifié, Fuhrmann relie donc l'intolérance à l'égard d'un peuple non élu et dénonce, 
sans doute dans une perspective pacifiste, l'hostilité séculaire des chrétiens envers les non-chrétiens.

Le motif du sacrifice sert ici également à accentuer l'opposition entre un Caïn lucide et un Abel naïvement, voire stupidement conformiste, dont la foi est un acte assez formel et dicté par l'habitude $(\mathrm{F}, 52)$; à son frère qui vient de sacrifier un agneau, Caïn demande : «Warum opferst du?», et Abel laisse alors reconnaître un certain utilitarisme prosaïque: «Es hilft manchmal» $(\mathrm{F}, 51)$, c'est-à-dire que cela permet d'avoir éventuellement de plus beaux moutons; avec 1'Abel de Fuhrmann, on est passé de la ferveur religieuse à la superstition obscurantiste. Jusqu'à la fin de la pièce, Caïn veut des certitudes alors qu'Abel se contente de croire, sans réfléchir (F, 49 et 51). Chez Weber, plus encore chez Fuhrmann, on ne peut dire si la "vraie» foi est du côté de Caïn, mais il est sûr qu'elle n'est plus du côté d'Abel.

11 La première partie de la pièce confronte deux conceptions antithétiques de l'utilité, celle de Caïn et celle d'Abel. Ce dernier voit en Caïn un parasite et un oisif sans ambition (F, 5 et 8), tandis que Caïn accuse de cupidité myope et d'étroitesse d'esprit ce frère qui, privé de tout sens poétique (en cela différent des Abel du XVIII ${ }^{\mathrm{e}}$ siècle), ne s'intéresse qu'à ce qui est matériel $(F, 7)$.

Quand Caïn entrevoit la possibilité de se consacrer à une entreprise de grande envergure (la mise en valeur du désert), il se met à travailler avec ardeur. Ses premiers succès (acte II) le font apparaître comme un homo faber talentueux, mais bien vite il échoue (F, 49).

13 Fuhrmann a clairement renversé les rôles traditionnels des deux frères. C'est désormais Caïn qui se sent à l'unisson d'une création qu'il respecte $(\mathrm{F}, 7)$; après avoir localisé l'Eden, il veut prendre modèle sur ce qu'il voit alors qu'Abel envisagerait de conquérir le paradis terrestre par la force (F, 10 et 13). Jusqu'au meurtre, c'est Caïn qui respecte les décisions divines. Il n'est pas non plus le Selbsthelfer individualiste habituel: soucieux des siens, il pense que Dieu a guidé ses pas vers le paradis pour lui permettre de libérer sa famille de la malédiction $(F, 10)$ et ne cesse de répéter qu'il ne réussira que si Dieu lui en donne la force. Le doute ne s'insinuera dans son esprit que quand il ne parviendra pas à irriguer son Éden; la découverte de l'arbre de vie lui redonnera confiance. Caïn tue Abel quand celui-ci déracine par infantilisme l'arbre de vie.

14 A la fin de la pièce, Caïn escalade une montagne, demande pardon à Dieu pour ses actions et ses blasphèmes (F, 86), puis arrivé au sommet, il s'écrie : «Kein Paradies!...», avant de tomber dans le vide (référence intertextuelle aux Kain de Kastropp et Weber où il se suicidait en sautant du haut d'une montagne). Son exclamation semble vouloir dissuader les hommes de tendre vers l'inaccessible, toujours plus beau dans l'imagination que dans la réalité (F, 37 et 39), ainsi que tend à le prouver le fait que Caïn, qui déjà se languissait de Lia dans la solitude de son Eden $(F, 31)$, ait demandé à cette dernière de dire au monde entier que le paradis n'existait pas. L'espoir constitue un danger pour l'humanité.

15 Comme au XVIII siècle, le sentiment, en particulier l'amour de Caïn pour sa sœur, occupe encore une place centrale chez Kastropp, Weber et Fuhrmann, et sous une forme modifiée chez Borngräber. Toutefois, avec Fuhrmann, une nouvelle perspective s'ajoute : Caïn n'est plus le fils qu'on croit insensible mais le fils qu'on dit paresseux. Déjà l'Adahm de Borngräber déclarait à Kajin, qui voulait dormir pour oublier le mal de vivre, que seul le travail permettait de desserrer l'étau de la vie (B, 13). Avec Fuhrmann, le renversement amorcé par Borngräber est total. Jusqu'alors, Caïn, sensible ou non, 
avait été un destructeur; maintenant, il s'efforce d'être un bâtisseur, comme si Fuhrmann épousait la perspective de Michelet qui en 1864 dénonce une injustice propre au « dieu des Juifs » qui « choisit l'oisif Abel contre le travailleur Caïn $»^{12}$. Il semble même que son ardeur au travail soit la forme sublimée de ce qui fut la révolte de ses prédécesseurs et que Leconte de Lisle (dans son poème "Qaïn ») et Baudelaire (poème "Le reniement de saint Pierre ») avaient poussée à son paroxysme.

Le motif du travail, préoccupation majeure de la société du XIX ${ }^{e}$ siècle, n'a pas seulement investi une partie du champ du sentiment; il s'est largement substitué aussi au motif byronien de la quête de la connaissance. A la problématique théologicométaphysique du travail comme punition qui occupait encore une position centrale chez Byron (II, 2, v. 125) est substituée une conception largement sécularisée de l'idée de travail. Mais tout comme la sensibilité ne permettait pas aux Caïn du XVIII ${ }^{e}$ siècle d'avoir une action positive, les efforts infructueux du Caïn de Fuhrmann montrent d'abord l'inaptitude de l'homme à transformer le monde par sa seule force. Lia, que Caïn aime, décèle le péché d'orgueil dans le fait que son frère, en qui sa famille voit un «Himmelstürmer» $(\mathrm{F}, 32)$, ait tenté de se substituer à Dieu en cherchant à forger son destin $(F, 73)$. Puis elle persuade son frère de ne pas se suicider et tente de lui montrer qu'il est possible de se méprendre sur les messages de la Providence. L'échec de Caïn dans son entreprise titanesque ne cautionne pas le robuste philistinisme d'Abel, mais il paraît comporter une critique implicite de l'esprit des Gründerjahre, voire de l'idée d'appropriation matérialiste du monde par le travail (B, 34). Chez Fuhrmann comme chez Byron, l'idéal poursuivi, pour louables qu'en soient les fondements, sort vaincu d'une confrontation avec la réalité qu'il prétendait modifier.

Avant Borngräber, la Providence intervenait objectivement dans l'action; chez Fuhrmann, elle n'apparaît plus que dans les propos des personnages et de surcroît réifiée en obstacle contre lequel la volonté humaine se brise. Chez Koffka, la sécularisation progresse encore. Malgré une citation de la Genèse en exergue, on n'est pas dans un univers du merveilleux ou de l'exceptionnel, mais dans une famille paysanne dont les membres paraissent porter comme par hasard les noms des héros bibliques.

Du point de vue formel, le Kain de Friedrich Koffka, rédigé en pleine guerre (1917), se distingue de tous les textes précédents par sa concision (un acte) et par le rejet du pathétique et du néoclassicisme ${ }^{13}$. Beaucoup d'autres expressionnistes ont tiré, par choix anti-naturaliste, une part importante de leur inspiration de la mythologie antique mais aussi de la Bible. Dans ce choix thématique, W. Rothe a vu une radicalisation existentielle du christianisme qui s'accentue après la première Guerre Mondiale et se traduit par un retour aux topoï primitifs : la mort, la souffrance, la pauvreté ${ }^{14}$. Toutefois, même quand le personnage principal est un héros biblique, on rencontre souvent des éléments grotesques, nombreux dans la littérature à partir des années 1890 et en relation avec l'idée de décadence ${ }^{15}$ : exemple prototypique, le Mathusalem d'Iwan Göll (Methusalem oder der ewige Bürger, 1922) semble sorti tout droit d'un tableau de Grosz. Comme chez Borngräber, le burlesque a relayé le merveilleux qui dans Der Tod Abels de Geßner était assuré par un démon.

Le drame de Koffka se présente comme un triptyque en prose, une longue conversation entre Caïn et Abel, encadrée par deux courtes scènes. Dans la scène liminaire, Adam reproche violemment à Caïn son oisiveté à laquelle il oppose l'extrême ardeur au travail d'Abel. Une objection formulée par Caïn («Man hat mich nicht not, wenn ich da 
bin», 0,713$)$ fait surgir un doute quant à la justesse de cette appréciation. Le thème biblique de Dieu refusant les offrandes de Caïn, rappelé dans la citation du Pentateuque en exergue, est totalement converti en un conflit à la justification incertaine entre le père et le fils. Adam bat son fils qui est défendu par sa mère : en cela, Koffka s'écarte de tous ses modèles, car les Adam antérieurs, à défaut de le comprendre, traitent toujours Caïn avec bienveillance.

Ce premier tableau met en place un double registre de langage : d'une part le langage quotidien d'un paysan soucieux du qu'en dira-t-on («man weist mit den Fingern auf dich und lacht über dich», O, 714), avec quelques arrière-plans naturalistes («Ich will fortgehen und noch einen Krug Bier holen auf den Abend» $(0,714)$, ainsi que la longue indication scénique qui ouvre le second tableau); d'autre part des réminiscences des accents tragiques qu'on trouve dans les textes antérieurs sur le thème de Caïn : «Hat er nicht Augen wie eines Tiers [...]?» (0, 714). Plus loin, Koffka ajoutera un embrayeur traditionnel du tragique, avec le motif de l'événement catastrophique qui est à la fois signe de la prédestination et signe avant-coureur du drame : «Als ich [Kain] geboren wurde, schlug drei Häuser weiter der Blitz in einen Stall. Die Mutter schrie, sie lag lange Tage mit bleichen Lippen wie tot» $(0,725)$.

$21 \mathrm{Au}$ cours du second tableau, le dialogue des deux frères révèle leurs véritables personnalités. Abel apparait d'abord sous les traits d'un rude paysan qui abat vaillamment son bétail avec un réel plaisir sans éprouver la moindre pitié pour l'animal alors que Caïn est effrayé par la vue du sang $(0,715)$. Ce motif de l'animal qu'on tue a une double fonction: d'une part, il est le signe de l'insensibilité d'Abel, et il apparaîtra plus tard que cette insensibilité, comme dans le cas de l'Adam de Fuhrmann, devient parfois cruauté à l'égard des autres; d'autre part, il souligne la désacralisation du motif, car il ne s'agit plus de sacrifices rituels (comme encore chez Fuhrmann) mais de découpages de quartiers de viande (d'ailleurs de veau et non plus d'agneau) comme dans l'arrière-boutique d'une boucherie.

Alors que Caïn est un être tourmenté, en cela conforme à l'image habituelle du "Grübler», qui gémit, parle et crie durant son sommeil, Abel énonce les poncifs d'un esprit qui se veut positif : «Ich schlafe, oder ich wache; dazwischen kenne ich nichts» $(0,717)$. Quand Caïn lui confie qu'il souffre d'insomnies, Abel lance une vérité de philosophe de Café du Commerce, sorte d'écho lointain et affadi de l'idée de rédemption par le travail: «Du solltest, wie ich, den Tag über arbeiten und dich ermüden. Du würdest fest schlafen in der Nacht und keine Träume mehr haben. Viele sagten mir, daß es keine Träume gebe im festen Schlaf» $(0,717)$.

Puis la conversation s'oriente vers un autre sujet : Abel parle à son frère d'une certaine Thirza (qui dans les textes du XVIII siècle est sa sœur). Abel, adolescent caricatural, rit grassement quand il est question d'amour. Caïn, qui semble aimer Thirza sans en être pleinement conscient, change brusquement de ton. Il sera désormais tantôt violent ou autoritaire, tantôt suppliant. Il commence par reprocher à son frère de torturer Thirza et emploie l'image du chevreuil traqué par les chiens (une image présente, dans un contexte différent, chez Kastropp et Weber). Le caractère d'Abel se précise et Caïn lui lance : «Es macht dir Freude, sie so zu hetzen mit deinen Spaßen? Sie will nicht lachen [...], aber du zwingst sie, zu lachen [...]» $(0,718)$. Cette saute d'humeur subite commence à inquiéter Abel, et quand l'hypersensible Caïn lui révèle qu'il l'a vu en rêve se fracasser le crâne sur un grosse pierre en plongeant dans l'eau d'une rivière, Abel lui demande affolé : «Was willst du von mir? Was weißt du von mir?» $(0,719)$. 

le transfert symbolique du «signe de Caïn ». Caïn se fait préciser à quel endroit précis $\mathrm{du}$ front de l'animal il faut frapper pour l'assommer. Le signe de Caïn est reporté du bourreau sur sa victime et ici le bourreau est Abel. Le motif de la blessure au front revient dans la bouche de Caïn d'une manière quasi obsessionnelle, mais il est toujours rapporté à Abel, soit que celui-ci l'inflige aux veaux, soit qu'il risque de se la faire en heurtant une pierre quand il plonge dans la rivière. Caïn s'exclame au comble de l'exaltation: «Hast du nicht Angst, du könntest die Stirn zerschmettern an diesem Stein?» $(0,719)$.

Un autre passage oppose un Caïn, âme bucolique qui observe les lis blancs de la prairie, à un Abel qui les écrase de son pied parce qu'il ne les voit pas, ce qui provoque chez Caïn un accès de colère assez infantile : «Warum hast du die Blume zertreten? Warum hast du sie mir zertreten, die Blume?» $(0,719)$. Abel lui répond comme à un malade ou à un enfant capricieux : «Verzeih, mir, Kain. Ich habe nicht gewußt, daß es deine Blume war» (ibid.). Au premier niveau d'une symbolique non développée, Abel détruit la pureté que Caïn défend. Mais dans le même temps le caractère névrosé de Caïn apparaît de plus en plus nettement. D'autres répliques révéleront son caractère phobique et son sentiment de culpabilité, qui l'opposeront un peu plus à Abel qui ne connaît aucune angoisse.

Abel se distrait en passant ses soirées au café en compagnie de quelques jeunes gens qui boivent beaucoup et courtisent sans raffinement la fille de l'aubergiste. Décrite avec une précision toute naturaliste, la vulgarité de ces médiocres grossiers, répliques lamentables des stratèges de «Ratskeller» que peint Heinrich Mann dans Der Untertan, se mue parfois en bêtise cruelle: un jour, pour plaisanter, ils humilient un jeune mendiant, une plaisanterie que Caïn, qui joue ici le rôle de la conscience lucide, trouve plus que douteuse. Cet épisode exprime métonymiquement l'hostilité instinctive et tendanciellement xénophobe des hommes comme Abel à l'égard de celui qui est autre. Ici Koffka reprend un motif déjà présent chez Fuhrmann mais en en faisant un des axes principaux du texte.

Peu à peu, nous apprenons qu'Abel fréquente quasi quotidiennement ce groupe et que Caïn l'épie avec une égale régularité, mû par un amour fraternel teinté de voyeurisme. Caïn a pu reconnaître ainsi la soumission servile que son frère témoigne au riche Akim. La violence de Caïn apparait de plus en plus distinctement, en particulier quand il oblige son frère à poser sa main sur la table pour constater qu'elle est puissante et belle alors que la sienne est « noiraude et tordue » $(0,723)$. Après ce qui peut sembler être le désir d'un psychopathe, l'admiration de Caïn pour son frère tend à se muer en ironie : «Aber was ist, mein lieber, gütiger Bruder? [...] du wärest, ich weiß, viel lieber allein im dunkeln Zimmer als im erhellten mit mir -» $(0,723)$, suggérant l'idée d'un Abel sournois.

Le drame de Koffka se joue à huis clos ; aucun événement extérieur ne vient en infléchir l'action: seuls les propos tenus engendrent les renversements d'attitude; les étapes et les rebondissements du dialogue remplacent les péripéties du drame d'action et énoncent la prépondérance du moi sur l'événement. Etant donné que Caïn sait, pour l'avoir observé dans le passé, tout ce que son frère lui révèle, sa haine semble naître intégralement de la verbalisation de ce qu'il a observé; cette verbalisation paraît entraîner une prise de conscience progressive de l'inimitié qui l'oppose à son frère et que rien, initialement, ne permettait de soupçonner. Il se pourrait y avoir à l'arrière- 
plan du texte de Koffka, qui semble réinterpréter à la lumière des savoirs psychiatriques de son temps la représentation traditionnelle au XVIII ${ }^{e}$ siècle d'un Caïn mélancolique, une connaissance des théories freudiennes et du rôle que celles-ci assignent au langage dans les processus psychiques. Que la folie furieuse de Caïn ne soit pas dérivée directement de l'observation des agissements d'Abel, mais de la confirmation que celui-ci apporte à Caïn de l'exactitude de ce qu'il a observé, montre que Koffka assigne au langage une efficacité qui fait défaut à la simple observation comme instrument de prise de conscience d'une réalité occultée par l'apparence : en d'autres termes, la réalité élaborée par le discours prime celle que dévoile l'observation. C'est la prise de conscience par le langage et non l'observation qui va amener Caïn à tuer.

On comprend peu à peu que Caïn a une obsession, qu'on peut mettre en relation avec le fait qu'Adam lui présente sans cesse son frère comme modèle : il voudrait être comme Abel : «Ich wünsche in jeder Stunde des Tages und der Nacht, daß ich wäre wie du, und tun könnte, was du tust!» $(0,726)$. Mais comme il est de plus en plus conscient du caractère peu admirable d'Abel, la folie résulte de cette contradiction. Elle prend naissance au moment où Caïn affirme : «Er [Adam] schlägt mich [...] und würde mich nicht schlagen, wenn du nicht da wärest als mein Bruder!» (ibid.). Le sentiment de tout ce qui le différencie d'Abel finalement l'emporte: «Hebe dich weg von meinen Augen, ich habe mit dir nichts gemein! Ich hasse dich!» (ibid.). Caïn se saisit alors d'une hache et, obsédé par le fait que les veaux aient les yeux braqués sur Abel quand celui-ci s'apprête à les tuer, il le tue en s'écriant : «Deine Augen, Abel!» $(0,727)$. Commis dans un moment de démence, cet acte s'inscrit finalement dans la logique du désir de Caïn d'être comme Abel, donc de tuer comme son frère.

Comme dans la quasi-totalité des textes de même thème, Caïn est saisi de graves remords sitôt le meurtre accompli. Mais ici son repentir le conduit à évacuer d'une phrase la réalité pourtant irréfutable que le dialogue a permis d'établir, et il s'écrie: «Mutter, erschlagen haben ihn meine Hände, Abel, der freundlich war und ein lieber Bruder zu mir. Abel, mein lieber Bruder, tot liegt er, welcher mir Gutes tat, ihn erschlug ich» $(0,728)$. Cette phrase prolonge le balancement que Koffka a maintenu pendant toute sa pièce entre les deux sentiments que Caïn éprouve pour son frère : la haine qui s'empare de lui fait partie d'un rapport amour-haine qui semble signifier qu'il aime son frère pour ce qu'il paraît, et qu'il le hait pour ce qu'il est. Le drame vécu par Caïn est la découverte progressive de la distorsion entre la réalité et l'apparence ; même une fois la réalité découverte, il ne cesse d'être tiraillé entre la répulsion qu'elle lui inspire et le désir de continuer de croire en la réalité de l'apparence : le désir, l'illusion volontaire, l'imaginaire priment le savoir. Le balancement de Caïn entre sa quête de la vérité et son attachement à l'apparence, cette crispation sur la volonté de croire en ce qu'il sait faux, et qui fonde sa folie plus qu'elle n'en est la conséquence, n'excluent pas la reconnaissance lucide de la vocation à l'errance éternelle de celui dont le moi est séparé de la conscience collective. Le fait qu'il prenne lui-même la décision de son propre bannissement suggère qu'il se soustrait à la justice des hommes pour ne se soumettre qu'à celle de Dieu. Mais par cette auto-condamnation, qui également justifie Abel et les siens, il assume son identité d'exclu qu'Adam et la société ont fait de lui et n'accusera pas expressément ce monde qui le rejette. Comme chez Borngräber, le Caïn de Koffka assume pleinement sa condition de criminel, mais dans la solitude parmi ses semblables, alors que tous ses devanciers avaient envisagé soit l'anéantissement par le suicide (chez Kastropp et Weber), soit l'attente du châtiment (chez Fuhrmann, il prie la 
veuve d'Abel de le tuer). Koffka peint, non pas une liberté individuelle, mais une absolue solitude.

31 Borngräber, Fuhrmann et Koffka ont recentré le thème de Caïn et Abel sur le conflit père / fils. La conception du rôle quasi éducateur des enfants chez Borngräber et Fuhrmann paraît emprunter des perspectives aux mouvements de réforme pédagogique du début du XX $\mathrm{XX}^{\mathrm{e}}$ siècle qui hypostasient l'enfant et dont le père castrateur freudien est certes l'exemple le plus connu, mais non le seul, ni moins encore le plus violent ${ }^{16}$. Dès avant cette époque, de nombreux textes en particulier de Sudermann, Hauptmann, Schnitzler, ou la pièce Der Sohn (1913) de Walter Hasenclever, thématisent des conflits parfois très violents entre les pères et les fils ${ }^{17}$. En 1913, «die Formel vom Hohen Meißner» proclame l'incompétence des pères et revendique le droit de la jeunesse à s'affranchir des tutelles: «Die Freideutsche Jugend will ihr Leben nach eigener Bestimmung, vor eigener Verantwortung, in innerer Wahrhaftigkeit gestalten» ${ }^{18}$. Koffka se distingue de Borngräber et Fuhrmann, car la démythification d'Abel et ses amis s'apparente chez lui à une critique des thèses qui proclament l'excellence de la génération nouvelle, de même que son autre pièce, Herr Oluf (1919), met en scène une sorte de «Burschenschaft» sans doute un peu moins grossière que le groupe des amis d'Abel mais au moins aussi violente.

La conversation d'Abel avec Caïn permet de dévoiler le peu-pensant dissimulé derrière le bien-pensant et le soi-disant fils-modèle, qui se trouve replacé dans son univers de médiocres violents. Ceux-ci représentent la société qui rend possible la niaiserie sûre et fière d'elle-même d'Abel et fait de Caïn un psychopathe. Comme dans les textes qui au moins depuis Geßner s'appuient sur le récit biblique de Caïn et Abel, Koffka tire parti du manichéisme que ce thème peut évoquer chez le spectateur pour tourner le dos à toute peinture en noir et blanc.

Dans les années 1910-20, d'autres écrivains reprennent des thèmes voisins, radicalisent certains traits, mais sans la complexité antithétique dont ont fait preuve Fuhrmann et Koffka. En face du rebelle prométhéen de Wilhelm Lennemann (1923), Anton Wildgans renouera dans Kain (1921) avec le manichéisme en accentuant toutefois le pessimisme : son Caïn, ivre de violence ${ }^{19}$, est le premier d'une lignée qui fera régner la haine dans le monde, tandis qu'Abel, un être certes harmonieux et poète habile, paraît bien superficiel. Si le Caïn de Wildgans « retombe » dans la violence et que sa réhabilitation ipso facto régresse, Abel n'en redevient pas pour autant clairement positif.

Un pessimisme dont ni Geßner ni Byron n'offraient d'exemple domine les textes de Fuhrmann et Koffka qui ne semblent pas entrevoir la possibilité d'existence d'un homme capable d'agir positivement sur le cours des choses, ni moins encore de parvenir au bonheur, sauf peut-être dans et par la bêtise. Tous deux, aussi bien que Borngräber, paraissent également concentrer leur attention sur l'immanence. Si Borngräber s'en tient à ce niveau, Fuhrmann laisse entrevoir qu'il rejette la perspective matérialiste d'une appropriation du monde par le seul travail. Alors qu'une lecture strictement immanente de la pièce de Koffka accréditerait volontiers, du fait de la désacralisation radicale qui la traverse, une exclusion de toute transcendance, ses articles révèlent en fait une perspective tout autre. Selon Koffka, qui critique la psychologie clinique du XIX ${ }^{e}$ siècle incapable selon lui d'expliquer l'homme pour la simple raison qu'elle le considère comme fini (alors que les comportements exceptionnels des grands héros shakespeariens révèlent qu'il est infini), le nouveau héros tragique à la manière de Shakespeare est l'homme moderne voué à l'inquiétude 
et à l'errance dans un monde matérialiste, parce qu'il est descendu dans le bourbier de la perte du sens, «in den Pfuhl der schändlichsten seelischen Verödung und der erbärmlichsten Verlassenheit von Geist und von Gott» $»^{20}$. Le thème de Caïn et Abel se trouve ainsi chez lui désacralisé et resacralisé.

\section{NOTES}

1. - Cf. G. Laudin, «Caïn au XVIII ${ }^{\mathrm{e}}$ siècle. Discontinuité tragique ou arbitraire des lois successorales? ", in : Études Germaniques, 1990/3, p. 273-289.

2. - On en trouve une liste relativement complète dans l'article «Kain und Abel» d'E. Frenzel, Stoffe der Weltliteratur, 1970, nombreuses rééditions.

3. - Gustav Kastropp, Kain, Stuttgart 1880, 376 p. <K>.

4. - Ludwig Weber, Kain. Ein Drama in 5 Akten und einem Vorspiel, Berlin 1896, 220 p. <W>.

5. - Ideen zur Philosophie der Geschichte der Menschheit, éd. Suphan, t. XIII, p. 431.

6. - Borngräber attache une grande importance à l'accentuation et à l'orthographe correctes des noms propres; on a ainsi : Adahm, Chawa, Chabel et Kajin. $-<B>$.

7. - L'idée de relations incestueuses entre Eve et ses fils se rencontre déjà chez Eutychius d'Alexandrie, cf. Bayle, Dictionnaire historique et critique, 1694, art. « Abel », note F.

8. - Par exemple de Das Mutterrecht 1861) de J.-J. Bachofen, ou encore de l'Ancient Society 1872) de Lewis H. Morgan, dont une traduction allemande a paru quelques années auparavant (Die Urgeschichte, Stuttgart, 1891).

9. - Otto Rank, Das Inzest-Motiv in Dichtung und Sage, Leipzig u. Wien 1912, p. 660-670, mentionne en particulier : Willy Speyer (Edipus, 1907), Klara Viebig («Frühlingsnot», Die Zeit du 26/05/1912 et Einer Mutter Sohn, 1906), J. Wassermann («Schicksal spielen», Oster-Zeit du 16/04/1911).

10. - Ibid., p. 657.

11. - Paul L. Fuhrmann, Kain. Tragödie in drei Aufzügen, R. Piper \& Co Verlag, 1909 ; <F>.

12. - Jules Michelet, Bible de l'humanité, Paris $1864^{3}$, p. 374-375.

13. - Présenté pour la première fois à Berlin le 09/0671918 par le théâtre « Das junge Deutschland " de Reinhardt qui a déjà joué ou jouera des pièces de Kokoschka, E. Lasker-Schüler, R. Sorge. Cité d'après l'anthologie de Karl Otten, Schrei und Bekenntnis. Expressionistisches Theater, Neuwied und Spandau 1959, $19622<0>$.

14. - Cf. art. sur Barlach, in: Christliche Dichter im 20. Jh. Beiträge zur europäischen Literatur, hg. von Otto Mann, Bern u. München 1968² , p. 269-285.

15. - A. Soergel U.C. Hohoff, Dichtung und Dichter der Zeit. Vom Naturalismus bis zur Gegenwart, $1963^{2}$, t. II, p. 48.

16. - Lothar Schlickert, «Geistige Väter und Söhne. Beobachtungen zum Wandel pädagogischer Autorität», in: Hubertus Teilenbach (Hg.), Das Vaterbild im Abendland, Stuttgart etc. 1978, t.I, p. 132.

17. - Cf. Kurt Wais, Das Vater-Sohn-Motiv in der Dichtung, Bd. 2 : 1880-1930, Berlin u. Leipzig 1931.

18. - Cité par L. Schuckert, art. cité, p. 135 et 207.

19. - «Gewalt! Gewalt! / Hahoiah, Gewalt! / Des Leuen Trunkenheit, / Zornmut des Starken, / Erbarmungslos! / Gewalt! Gewalt! / [...] Und auf der Demut Nacken / Setzt er die Ferse / Unter Weltengelächter», in: Sämtliche Werke, Bd. 4, Wien u. Salzburg, p. 314. 
20. - Koffka, «Über Shakespeare und die Wiedergeburt des Tragischen», in: Neue Rundschau, 1921 (Nachdruck Königstein/Ts. 1980), p. 1046-1068, ici p. 1057.

\section{RÉSUMÉS}

Publiés entre 1908 et 1917, les textes de Borngräber, Fuhrmann et Koffka s'inscrivent dans le prolongement d'une longue série de mutations qui ont affecté dès le XVI ${ }^{\mathrm{e}}$ siècle le traitement littéraire du thème de Caïn et Abel dans la littérature allemande. Comme déjà plusieurs auteurs du XVIII ${ }^{\mathrm{e}}$ siècle, ils l'investissent de préoccupations et valeurs sociales de leur temps, les relations familiales et le travail. Si Borngräber en donne une lecture avant tout mythocritique en faisant ressortir un érotisme primitiviste, Fuhrmann et Koffka poursuivent la réhabilitation partielle de Caïn, engagée dès les années 1760, mais surtout accentuent la « démolition » du héros Abel, déjà amorcée par Byron et des auteurs allemands de la fin du XIX ${ }^{e}$ siècle. Autrefois pieux, docile et obéissant, Abel est chez eux naïvement conformiste, hypocrite, misogyne. Inversement, Caïn est chez Fuhrmann l'homo faber que déjà Michelet discernait en lui ; toutefois, malgré ses efforts, il est, chez Fuhrmann comme chez Koffka, tenu par les siens pour paresseux, tout comme son ancêtre du XVIII ${ }^{\mathrm{e}}$ siècle était considéré à tort comme insensible.

Koffka, pour qui le nouveau héros tragique est l'homme moderne à l'identité aliénée et voué à l'inquiétude dans un monde matérialiste, pousse la désacralisation plus loin que ses devanciers: au sacrifice est substituée une prosaïque métonymie de la violence, l'abattage de veaux par un Abel qui fréquente les cafés dans une sorte de rite quotidien. Le bien-pensant que son père voit en lui révèle progressivement sa véritable nature de peu-pensant que Caïn, personnage tourmenté, psychotique et infantile, finira par tuer, car il lui faut égaler ce frère que son père lui présente comme modèle.

Die zwischen 1908 und 1917 erschienenen Texte von Borngräber, Fuhrmann und Koffka führen den bereits seit dem 16. Jahrhundert in der deutschen Literatur vielfältig modifizierten Kainund-Abel-Stoff fort. Ähnlich wie schon mehrere Autoren im 18. Jahrhundert behandeln sie ihn unter Berücksichtigung gewichtiger gesellschaftspolitischer Ideologerne ihrer Zeit wie Arbeit und Familie. Während Borngräber eine vor allem mythokritische Lesart entwickelt, die ihn eine primitive Erotik hervorheben läßt, verfolgen Fuhrmann und Koffka die bereits seit 1760 intendierte, teilweise Rehabilitierung von Kain, wobei sie aber vor allem die schon bei Byron und bei deutschen Autoren am Ende des 19. Jahrhunderts flüchtig begonnene «Zerstörung des Helden» Abel weiter ausbauen. Der ehemals fromme, gelehrige und gehorsame Abel wird bei ihnen zum naiven, scheinheiligen und frauenfeindlichen Konformisten. Kain hingegen wird bei Fuhrmann zum homo faber, den schon Michelet in ihm erkannte. Wie schon die Kain-Gestalten des 18. Jahrhunderts zu Unrecht als unsensibel galten, so genießt auch Kain bei Fuhrmann und Koffka, obwohl arbeitsam, in der Familie den Ruf eines Faulpelzes.

Koffka, der im modernen, seiner Identität entfremdeten und in einer materialistischen Welt rastlosen Menschen den neuen tragischen Helden wahrnehmen möchte, treibt die Entsakralisierung noch weiter auf die Spitze als seine Vorgänger: anstelle des Opfers setzt er eine nüchterne Metonymie der Gewalt, nämlich das Abschlachten der Kälber durch einen Abel, dessen regelmäßige Kneipenbesuche zum alltäglichen Ritual werden. Der Konformist, den sein Vater in ihm sieht, entpuppt sich allmählch als Hohlkopf, den der psychotische, selbstquälerische Kain 
schließlich ermordet, damit er dem aus Sicht des Vaters vorbildlichen Bruder ähnlich werden kann.

\section{AUTEUR}

GÉRARD LAUDIN

Université de Rouen 